\title{
Cystic Fibrosis: The Prognosis for Five-year Survival
}

\author{
JAMES D. KNOKE, ${ }^{(17)}$ ROBERT C. STERN, CARL F. DOERSHUK, THOMAS F. BOAT, AND \\ LEROY W. MATTHEWS \\ Departments of Biometry and Pediatrics, Case Western Reserve University, Cleveland, Ohio, USA
}

\begin{abstract}
Summary
Statistical discriminant analysis is applied to 41 concomitant variables obtained during the first year of study of $\mathbf{2 2 6}$ patients with cystic fibrosis. A discriminant function based on six variables is developed which can be used as a predictive index. This index estimates the probability of 5-year survival for an individual patient and can also be used to classify patients into one of two groups: (1) will live for 5 years or (2) will die within 5 years.

\section{Speculation}

A classification rule for cystic fibrosis is presented which correctly assigns $90 \%$ of the 5-year survivors and $84 \%$ of those who expire. Such a classification scheme is useful for clinical and research purposes.
\end{abstract}

An index that assesses the severity of a chronic disease in an individual patient is frequently useful. Clinical indices for cystic fibrosis have previously been devised $(13,15)$. This study applies statistical discriminant analysis to longitudinal data on 226 patients to obtain an index predictive of five year survival.

Discriminant analysis is a multivariate statistical method for predicting a categoric response variable (e.g., survived or expired) from a collection of concomitant variables (6). A discriminant function is estimated from a sample of patients on whom both the response variable and the concomitant variables are available. The discriminant function along with the values of the concomitant variables can then be used for prediction, that is, to estimate a new patient's probability of survival or to classify a new patient into one of two groups: (1) will live or (2) will die. Statistical discriminant analysis has previously been used to predict the survival of patients with shock after myocardial infarction $(1,12)$ or barbiturate overdose (2) and of patients recovering from acute myocardial infarction (8).

\section{MATERIALS AND METHODS}

\section{STUDY SAMPLE}

All patients seen at the Cystic Fibrosis Center (Rainbow Babies and Childrens Hospital, Cleveland) between 1958 and 1964 were included in the study if they survived for at least 1 year and 5-year survival information was available. Patients referred after 1964 who survived at least 1 but less than 5 years were also included. With the exception of newer anti-pseudomonas antibiotics, there is no difference in the treatment of cystic fibrosis today from that employed in the 1958-1964 period $(9,14)$. Informed consent was obtained.

Cystic fibrosis was diagnosed by elevated sweat chloride along with either typical pulmonary or digestive findings or a family history of cystic fibrosis. One patient who died from accidental causes was excluded. The first year of data collection may not be the first year following diagnosis of cystic fibrosis, if the diagnosis was not made at this institution. In any event, the first year of data collection did not begin before the completion of the calendar quarter in which the diagnosis was made, to allow the patient to stabilize. Approximately $57 \%$ of the patients were less than 5 years of age, $86 \%$ less than 10 years, and $96 \%$ less than 15 years at the beginning of the first year of data collection.

Of the 226 patients in the study sample, 195 survived for 5 years from the middle of the first year of data collection. The proportion survived of the entire sample, $86.3 \%$, however, should not be taken as an estimate of the probability of 5-year survival of a randomly selected patient who has survived at least 1 year following diagnosis. Rather, this probability should be estimated by the proportion surviving among the patients included between 1958 and 1964; namely, 92.4\%.

\section{CONCOMITANT VARIABLES}

Sixty-one quantitative variables were obtained from the first year of medical data on all 226 patients. Additionally, the results of 10 pulmonary function (PF) tests were available for 148 patients. These 148 patients are referred to as the PF subsample. The 78 patients not in the PF subsample were too young to cooperate for PF testing during the first year of data collection. The 71 variables are detailed in the Appendix. A variable was included if it was available for all patients, might possibly be associated with mortality, and varied over an ordinal scale.

Thirty of the variables included reflect the treatment a patient received and thus only indirectly reflect his condition. These variables are not included in the predictive index but are given in the Appendix, where those which differ significantly between the survivors and the nonsurvivors are noted.

\section{METHODOLOGY OF DISCRIMINANT ANALYSIS}

Statistical discriminant analysis employs data on a sample of patients, here divided into groups, "surviving" and "expiring," based on their 5-year survival, to estimate the coefficients of a linear discriminant function. The discriminant function can then be employed predictively on a different group of patients, to estimate a patient's probability of survival and/or to classify patients into two groups, those most likely to survive and those most likely to die. These inferred groups are termed the "will live" and "will die" groups to distinguish them from the known surviving and expiring groups.

The first phase of a discriminant analysis is to select from the concomitant variables a minimal subset sufficient for discrimination. A forward stepwise analysis (4) was used to determine the approximate number of variables for the discriminant function, considering the overall significance of the difference between groups, the minimal significance of the individual variables, and the estimated probabilities of misclassification. The 
stepwise analysis, along with a univariate analysis of each variable, was also used to obtain a feasibly sized subset of variables (a maximum of about 18) for evaluating all possible discriminant functions (10). The best discrimination possible was sought while avoiding variables that, either statistically or practically, did not significantly improve the discrimination.

Having selected the variables to be included, estimates of the coefficients of the linear discriminant function are easily computed. The discriminant function evaluated for the data of a given patient is termed the "discriminant score" for that patient. This score can be used to estimate the probability of survival for 5 years and to assign the patient to the will live or will die group.

Finally, the accuracy of prediction of the discriminant function was assessed by estimating the probabilities of misclassification by the "leaving-one-out-method." Such estimates are less optimistically biased than those resulting from simple resubstitution of the sample values into the discriminant function (7).

Although nonlinear functions can be used in discriminant analysis, there was no a priori information to suggest that any particular family of mathematical functions relates condition to survival in cystic fibrosis more accurately than does a linear function. A linear analysis does have the advantage of relative ease of variable selection and numerical computation. It also has been shown that a linear analysis is frequently a good approximation to a potentially more optimal nonlinear analysis $(3,11)$. Finally, the results of the present analysis, regardless of optimality properties, have obvious clinical usefulness.

\section{RESULTS}

Of the 71 variables obtained during the first year, 45 differed significantly between the surviving and expiring groups when evaluated by an appropriate univariate statistical test. These 45 variables are identified in the Appendix.

A stepwise analysis of the 41 nontreatment variables, including the pulmonary function variables, was performed on the PF subsample. Although 9 of the $10 \mathrm{PF}$ variables were highly significant univariately, only 1 , maximum breathing capacity, exhibited any possibility of significantly improving the discrimination, given the other more predictive variables already in the function. The PF variables were therefore excluded and only the 31 nontreatment and non-PF variables were used in the subsequent analysis. This allowed the employment of the entire study sample, with the attendent benefits of a larger sample size and a broader age distribution.

The stepwise analysis using the 31 variables obtained during the first year and the entire study sample indicated that six variables should be included in the discriminant function. These variables are detailed in Table 1 . Given these six variables, the

Table 1. Variables in discriminant function

\begin{tabular}{|c|c|c|c|}
\hline \multirow[b]{2}{*}{ Symbol } & \multirow{2}{*}{ Variable $^{1}$} & \multicolumn{2}{|c|}{ Mean values } \\
\hline & & $\begin{array}{l}\text { Survived } \\
\text { group }\end{array}$ & $\begin{array}{l}\text { Expired } \\
\text { group }\end{array}$ \\
\hline $\mathrm{X}_{1}$ & $\begin{array}{l}\text { Proportion of cultures positive for } \\
\text { any pseudomonas }(26)^{2}\end{array}$ & 0.352 & 0.969 \\
\hline $\mathrm{X}_{2}$ & $\begin{array}{l}\text { Proportion of cultures positive for } \\
\text { Hemophilus influenzae }(30)\end{array}$ & 0.005 & 0.045 \\
\hline $\mathrm{X}_{3}$ & General well being score (9) & 22.9 & 17.5 \\
\hline $\mathrm{X}_{4}$ & $\begin{array}{l}\text { Proportion of cultures positive for } \\
\text { other pathogenic organisms ( } 31 \text { ) }\end{array}$ & 0.096 & 0.156 \\
\hline $\mathrm{X}_{5}$ & Chest roentgenogram score (12) & 17.6 & 9.0 \\
\hline $\mathrm{X}_{6}$ & $\begin{array}{l}\text { Proportion of cultures positive for } \\
\text { pseudomonas and Staphylococ- } \\
\text { cus aureus simultaneously (28) }\end{array}$ & 0.154 & 0.300 \\
\hline
\end{tabular}

${ }^{1}$ Variables are given in order of decreasing relative significance.

${ }^{2}$ Numbers in parentheses refer to variable numbers in the Appendix, Part III. addition of any other variable does not significantly improve the discrimination.

All possible discriminant functions were evaluated using the six variables given in Table 1 and 11 others suggested by the univariate or stepwise analyses as possibly being important for prediction. The 17 variables employed are identified in the Appendix. This analysis confirmed that six is the most reasonable number of variables to include, and that the set of variables selected by the stepwise analysis was indeed the best set of six. Additionally, this analysis failed to exhibit any other set of six variables, suboptimal but close to optimal, that might be preferable to the best six in terms of ease, cost, or reliability of collection.

The recommended discriminant function is $\mathrm{Y}=-3.65 \mathrm{X}_{1}-$ $25.4 \mathrm{X}_{2}+0.432 \mathrm{X}_{3}-4.72 \mathrm{X}_{4}+0.193 \mathrm{X}_{5}+2.84 \mathrm{X}_{6}$. If the discriminant function is evaluated for a particular patient, by substituting the $\mathrm{X}_{1}, \ldots, \mathrm{X}_{6}$ observed over 1 year, the value of $\mathrm{Y}$ obtained is that patient's discriminant score. The probability of 5-yr survival, $\mathrm{P}$, is given by: $\mathrm{P}=1 /\left(1+0.082 \mathrm{e}^{8.30-\mathrm{Y}}\right)$. The value of $P$ can equivalently be obtained by reference to Figure 1 .

The sign of the coefficient for $\mathrm{X}_{6}$ is positive while the signs for $\mathrm{X}_{1}, \mathrm{X}_{2}$, and $\mathrm{X}_{4}$ are negative. Thus, although occurrences of pseudomonas, Hemophilus influenzae, and other pathogenic organisms are unfavorable to a prognosis, the occurrence of Staphylococcus aureus with pseudomonas is more favorable than the occurrence of pseudomonas alone. This is consistent with the supposition that as the severity of pseudomonas infection increases the staphylococci eventually either are unable to colonize or are no longer viable in sputum samples submitted for culture.

The classification rule that minimizes the total number of misclassifications is that which classifies a patient into the will live group if his probability of survival is greater than $50 \%$ and into the will die group otherwise. This rule results in an estimated probability of misclassifying as will die a patient who actually survives as: $\mathrm{P}(\mathrm{D} / \mathrm{S})=0.021$ or $2 \%$, and an estimated probability of misclassifying as will live a patient who actually expires as: $\mathrm{P}(\mathrm{L} / \mathrm{E})=0.452$ or $45 \%$. In a population where $92.4 \%$ will actually survive, the overall probability of misclassification is: $\mathrm{P}($ misclass $)=0.021(.924)+0.452(0.076)=0.053$ or $5.3 \%$.

Although the classification rule based on a $50 \%$ threshold minimizes the total number of misclassifications, an alternative rule which correctly classified a larger proportion of the expired group, at the expense of an increased number of misclassifications in the survived group and in the entire sample, may be

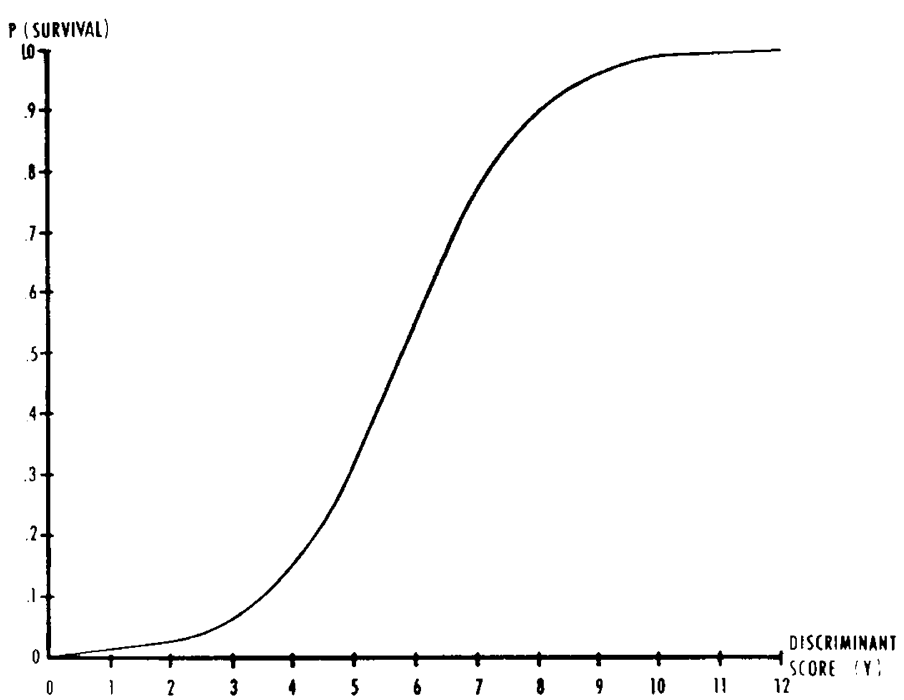

Fig. 1. Probability of 5-year survival as a function of discriminant score. 
more useful for clinical or research purposes. Such an alternative rule can be obtained by raising the threshold of estimated probability of survival that a patient must present to be classified into the will live group. For example, if patients are classified into the will live group only if their estimated probabilities of survival are at least $92.4 \%$, the resulting estimated probabilities of misclassification are: $\mathrm{P}(\mathrm{D} / \mathrm{S})=11 \%, \mathrm{P}(\mathrm{L} / \mathrm{E})=16 \%$, and $\mathrm{P}($ misclass $)=11.5 \%$.

The $92.4 \%$ threshold for the alternative classification rule was obtained by incorporating unequal "costs of misclassification" for the survived and expired groups, and then determining the rule which minimizes the total cost of misclassification. It was assumed that the cost of misclassifying a patient from the expired group was 12.2 times the cost of misclassifying a survived patient. The ratio 12.2 was chosen because this is the ratio of the expected number of surviving patients to the expected number expiring. Such a choice of costs results in an alternative rule which tends to equalize the proportions misclassified from the two groups.

It is apparent from the estimated probabilities of misclassification that, proportional to the total number of patients, it is more difficult to correctly identify those who will expire than those who will survive. Desirite an increased proportion of misclassifications overall, the alternative classification rule may be clinically useful when it is desired to identify a subset of patients at high risk of death for special treatment or study. Also, of the 21 survivors misclassified by the alternative rule, only 6 were considered by their physicians to be doing well at the end of the 5 years of follow-up. Of the other 15 , within 2 years after the end of the 5-year period, 5 had died and 10 remained in precarious health.

\section{DISCUSSION}

A discriminant function has several practical uses. The simple knowledge of what variables form a minimal set sufficient for prediction should be noteworthy. In this instance, the inclusion of the general well being and roentgenogram scores is of practical, clinical importance. The discriminant function can also be used to estimate the probability of 5-year survival for an individual patient, for evaluation and counseling. The function can additionally be used for research purposes to classify patients into groups more generally, e.g., the extreme groups of those with very good and very poor long term prognoses. A group with a very good long term prognosis might be desired for a
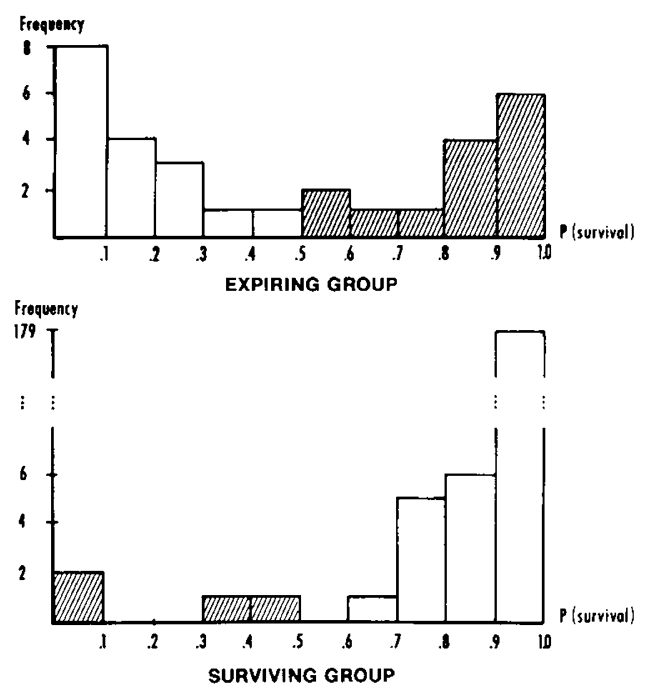

Fig. 2. Frequency distributions of probabilities of survival. The shaded portions indicate patients misclassified by the $50 \%$ threshold rule. longitudinal study, whereas a group with a very poor prognosis might be suitable for evaluating alternate treatments.

When the discriminant function is used for classification, the rule which classifies a patient into the will live group if his estimated probability of survival is greater than $50 \%$ minimizes the expected total number of misclassifications. For clinical applications, however, an alternative rule which correctly classifies a larger proportion of the expired group may be more useful. The probabilities of survival, by the leaving-one-out method, of our 226 patients are illustrated in Figure 2. Figure 2 allows the visualization of the two probabilities of misclassification resulting from any classification rule, although the misclassifications for the $50 \%$ threshold rule are illustrated. The difficulty of correctly predicting a larger proportion of the expired patients results from the U-shaped distribution of the probabilities of survival for patients who actually expire.

In performing the discriminant analysis only one set of concomitant variables was employed for each patient, to satisfy the assumption of independence required by the underlying statistical theory. For clinical prediction, however, it may be useful to estimate the probability of 5-year survival for each patient every year. Such repeated estimation is certainly reasonable as long as the patient remains less than 15 years of age.

\section{APPENDIX}

\section{CONCOMITANT VARIABLES FROM THE FIRST YEAR}

The symbol "*" indicates a difference between the surviving and expiring groups at significance level 0.05 , by a univariate test, whereas the symbol "**" indicates a difference at significance level 0.01 . When the concomitant variable was dichotomous, Pearson's chi square test (without correction for continuity) was used. Otherwise, Student's $t$ test was employed. The notation "(d)" denotes a dichotomous variable and "(p)" a polytomous, ordinally scored variable. The symbol " $\uparrow$ " identifies the 17 variables used in the all possible discriminant functions analysis. Complete descriptions of the variables are available from the authors.

I. Treatment variables

1. Type of aerosol therapy (p)

* 2. Frequency of aerosol therapy (p)

3. Frequency of postural drainage $(p)$

** 4. Type of mist tent (p)

5. Type of mist tent solution, I (p)

6. Type of mist tent solution, II (p)

7. Regularity of mist tent usage

8. Antihistamine used (d)

** 9. Anabolic steroid used (d)

Variables 10-30 are the number of weeks of usage of:

10. Penicillin

11. Novobiocin

**12. Tetracycline (including methacycline and doxycycline)

13. Erythromycin

**14. Chloramphenicol

**15. Ampicillin

$* * 16$. Sulfisoxazole

$* * 17$. Neomycin

$* * 18$. Oxacillin

${ }^{* *} 19$. Lincomycin

$* * 20$. Sulfisoxazole for pseudomonas

$* * 21$. Tetracycline for pseudomonas

${ }^{* *} 22$. Sulfisoxazole and tetracycline simultaneously for pseudomonas

**23. Chloramphenicol for pseudomonas

$* * 24$. Sulfisoxazole and tetracycline simultaneously for pseudomonas

**25. Ampicillin for pseudomonas

*26. Lincomycin for Staphylococcus aureus

27. Penicillin for Staphylococcus aureus 
**28. Oxacillin for Staphylococcus aureus

29. Chloramphenicol for Staphylococcus aureus

30. Erythromycin for Staphylococcus aureus

II. Pulmonary function test variables, best score during year

** 1. Actual vital capacity

** 2. Combined vital capacity

** 3. Ratio of forced expiratory volume in $1 \mathrm{sec}$ to vital capacity

** 4. Expiratory reserve volume

** 5. Inspiratory volume

** 6. Maximum breathing capacity

** 7. Functional residual capacity

** 8. Residual volume

9. Total lung capacity

**10. Ratio of residual volume to total lung capacity

III. Other variables

$\dagger^{* *}$ 1. Age at entry to care at this institution

2. $\operatorname{Sex}(d)$

3. Number of respiratory infections during the year

4. Decrease in appetite $(\mathrm{d})$

5. Nasal polyps (d)

$\dagger^{*}$ 6. Liver enlargement (including cirrhosis) (d)

7. Pneumothorax (d)

8. Cirrhosis (d)

$\dagger^{* *}$ 9. Best general well being score (case history score (5))

$\dagger * * 10$. Best physical exam score (pulmonary findings score (5))

**11. Best nutrition score (5)

$\dagger^{* *} 12$. Best chest roentgenogram score (5) (all scoring was done by the same radiologist)

*13. Age at beginning of study year

14. Number of days hospitalized during the study year

$\dagger * * 15$. Pulmonary flare up $(\mathrm{p})$

16. Standardized height gain

$\dagger^{* *} 17$. Standardized weight gain

18. Wheezing (d)

$\dagger^{* *} 19$. Coughing $(\mathrm{p})$

$\dagger * * 20 . \quad$ Hemoptysis $(\mathrm{p})$

$\dagger * * 21$. Rales (p)

$\dagger * * 22$. Clubbing (p)

23. Normal bowel movement (d)

The number of cultures taken varied among the patients and were summarized monthly. Thus the maximum number of cultures reported is 12 . A reasonably healthy patient will be cultured at 6-week intervals for a total of eight cultures. Some patients were cultured less than eight times, however, because of missed appointments. Variables 24-31 are the proportions of total cultures reported positive for: $\dagger * * 24$. Only normal flora

$\dagger^{* *} 25$. Any type of mucoid pseudomonas

$\dagger^{* *} 26$. Any type of pseudomonas

27. Staphylococcus aureus

$\dagger^{* *} 28$. Any type of pseudomonas and Staphylococcus aureus simultaneously

29. Streptococcus or pneumococcus

$+* * 30$. Hemophilus influenzae

$\dagger 31$. Other possibly pathogenic organisms, including proteus, klebsiella, Escherichia coli, and other gramnegative rods

\section{REFERENCES AND NOTES}

1. Afifi, A. A., Chang, P. C., Liu, V. Y., daLuz, P. L., Weil, M. H., and Shubin, H.: Prognostic indexes in acute myocardial infarction complicated by shock. Amer. J. Cardiol., 33: 826 (1974).

2. Afifi, A. A., Sacks, S. T., Liu, V. Y., Weil, M. H., and Shubin, H Accumulative prognostic index for patients with barbiturate, gluthethimide and meprobamate intoxication. N. Engl. J. Med., 285: 1497 (1971).

3. Chang, P. C., and Afifi, A. A.: Classification based on dichotomous and continuous variables. J. Amer. Statist. Ass., 69: 336 (1974).

4. Dixon, W. J., ed.: BMD: Biomedical Computer Programs, pp. 233-253 (University of California Press, Berkeley, 1974).

5. Doershuk, C. F., Matthews, L. W., Tucker, A. S., Nudelman, H., Eddy, G., and Wise, M.: A 5 year clinical evaluation of a therapeutic program for patients with cystic fibrosis. J. Pediat., 65: 677 (1964)

6. Lachenbruch, P. A.: Discriminant Analysis (Hafner Press, New York, 1975)

7. Lachenbruch, P. A., and Mickey, M. R.: Estimation of error rates in discriminant analysis. Technometrics, 10: 1 (1968).

8. Luria, M. H., Knoke, J. D., Margolia, R. M., Hendricks, F. H., and Kuplic, J. B.: Acute myocardial infarction: Prognosis after recovery. Ann. Intern. Med., 85: 561 (1976).

9. Matthews, L. W., Doershuk, C. F., Wise, M., Eddy, G., Nudelman, H., and Spector, S.: A therapeutic regimen for patients with cystic fibrosis. J. Pediat., 65: 558 (1964).

10. McCabe, G. P., Jr.: Computations for variable selection in discriminant analysis. Technometrics, 17: 103 (1975).

11. Moore, D. H., II: Evaluation of five discrimination procedures for binary variables. J. Amer. Statist. Ass., 68: 399 (1973).

12. Shubin, H., Afifi, A. A., Rand, W. M., and Weil, M. H.: Objective index of haemodynamic status for quantitation of severity and prognosis of shock complicating myocardial infarction. Cardovasc. Res., 2: 329 (1968).

13. Shwachman, H., and Kulczycki, L. L.: Long term study of one hundred five patients with cystic fibrosis. A. M. A. J. Dis. Child., 96: 6 (1958)

14. Stern, R. C., Boat, T. F., Doershuk, C. F., Tucker, A. S., Primiano, F. P. Jr., and Matthews, L. W.: Course of cystic fibrosis in 95 patients. J. Pediat., 89: 406 (1976)

15. Taussig, L. M., Kattwinkel, J., Friedewald, W. T., and di Sant'Agnese, P. A.: A new prognostic score and clinical evaluation system for cystic fibrosis. J. Pediat., 82: 380 (1973).

16. This research was partially supported by Grants AM-08305, HL-13885, and GM-12302 from the National Institutes of Health and grants from the Cleveland Cystic Fibrosis Foundation and the Health Fund of Greater Cleveland.

17. Requests for reprints should be addressed to: Dr. James D. Knoke, Department of Biometry, University Hospitals, Cleveland, OH 44106 (USA).

18. Received for publication May 5, 1977.

19. Accepted for publication August 16, 1977. 